\title{
Ready, aim, fire: legitimizing the gaming environment
}

\author{
M. McGlothlin \\ University of Florida, School of Architecture, Gainesville, Florida, USA
}

\begin{abstract}
The digital gaming industry, perhaps to the chagrin of the architectural community, has become the epitome of modern business success, drawing in remarkable profits while leading a quiet revolution in youth culture. The systems that have become available in the last several years are sophisticated enough to not simply hint at virtual reality, but rather establish a surprisingly convincing world into which the participant is immersed. These effects are so stimulating that individuals engaged in a first-person shooter combat arena respond physically to the digital simulations, ducking and crouching with comical evasiveness to the virtual projectiles being launched at them. Given the spatial realism being achieved, one would expect that the architectural profession, identified with the crafting of space, would readily embrace these technologies, but the opposite seems to be the case. These environments are viewed as distractions with little value beyond entertainment, with minimal significance in the domain of critical space. The on-going research attempts to bridge this theoretical gap between architectural discourses on spatial perception and the fascinations of virtual immersion. The simulations of digital gaming are too profound to be dismissed, offering tremendous potential as critiques of digital culture, as well as exposing the sensitive underbelly of societal norms relative to the digital interface. While in no way attempting to act as an apologist for the gaming industry or its fixation with the grotesque, a critical examination of its spatial character brings to light an array of architectural origins, both theoretical and spatial. To this end, the fundamental tenants of game space finds resonance, or perhaps dissonance, within the most cherished of architectural experiences, and perhaps begins to devalue architectural poetics by collapsing the importance of physical time and space, making spatial simultaneity possible within the virtual seductions of the screen.
\end{abstract}

Keywords: immersive environments, architectural representation, digital critique, labyrinths, Piranesi. 


\section{Introduction}

"Because what does not correspond to any mathematical law is the arrangement of the openings. Some rooms allow you to pass into several others, some into only one, and we must ask ourselves whether there are not rooms that do not allow you to go anywhere else. If you consider this aspect, plus the lack of light or of any clue that might be supplied by the position of the sun (and if you add the visions and the mirrors), you understand how the labyrinth can confuse anyone who goes through it, especially when he is already troubled by a sense of guilt. Remember, too, how desperate we were last night when we could no longer find our way. The maximum of confusion achieved with the maximum of order: it seems a sublime calculation. The builders of the library were great masters."

- Brother William of Baskerville: The Name of the Rose [1]

Mankind's reverent infatuation with labyrinthine space is long standing and complex, stretching the expanse of both time and distance. The relevance, if not necessity, of these twisting, fractured and disorienting spaces is so deeply imbedded within the human psyche that the conceptual basis is no more than rumination, reinvented with each subsequent generation as a new metaphorical veil, draped across an original spatial structure. The metaphors of the labyrinth have been chronologically transient, shifting with the cultural needs, whether those needs be theological, aesthetic or experiential. Yet it is the physical structure that has persisted intact, an underlying architectonic skeleton of space and form that provides the formal order, the spatial logic, and the rationale of occupation onto which, or from which, meaning can be derived. The labyrinth exists as series of dualisms, simultaneously a construct of physical and intellectual realms, informing and informed by the occupant, and shaping and shaped by the transcendental experience.

\section{Labyrinth as artefact}

This question of experience becomes integral to the perception of the labyrinth. Its historical significance within western traditions is bound within a number of theological/mythological braids, first offered within the well-tread stories of King Minos and the Minotaur. Based off of Ovid's version of the story, the origin of the Minotaur and its subsequent imprisonment within the labyrinth starts with Pasiphae, wife of the king, who, during the siege of Athens by Crete, falls in love with a bull, and with the assistance of the inventor Daedalus consummates the adulterous relationship, giving birth to the Minotaur. With the return of King Minos, who is infuriated with his wife's infidelity, Daedalus is commanded to construct the labyrinth, a maze so complex and disorienting that none who ventured into it would find escape (including Daedalus). Human offerings were made to the Minotaur, tributes of the defeated Athenians. One of 
these sacrifices was Theseus, son of King Aegeus, who had charmed King Minos' daughter, Ariadne. Ariadne, out of her love for the young Theseus, gave him two gifts to help him solve the labyrinth's spatial riddle and slay the Minotaur. The first gift was a ball of pitch, to be lodged in the Minotaur's mouth, and the second was a ball of thread, allowing Theseus to retrace his steps through the bewildering maze. Upon Thesues' escape, and subsequent retreat to Athens with Ariadne (whom he left en route at Naxos), King Minos imprisons Daedalus and his son Icarus within the labyrinth. Daedalus then crafts wings of feathers and wax for he and his son, by which they can escape the labyrinth. However, Icarus fails to heed his father's warnings of flying too close to the sun, and the wax on his wings melts and Icarus plummets to his death [2, pgs 11-13].

While the myth of the Minotaur is far more complex that this abbreviated version, and carries a number of variants, the basic premise of the physical labyrinth as an indecipherable and inextricable linkage of chambers and passage ways was geometrically codified (literally within the continuation of the myth with Daedalus crafting the labyrinthine story into the doors to the temple to Apollo at Cumae). It is this physical impression of the labyrinth, this canonical spatial palimpsest, which has proven the most enduring. Though there is a considerable lineage to the myth, and its transmutations into other similarly crafted narratives (such as Dante's inferno or the biblical accounts of the life, death and resurrection of Christ), the physical form has become an enduring symbol and spatial ordering device likened to the most basic levels of the claustrophobic confinements and redemptions.

This is quickly notable in the medieval labyrinths so often associated with the gothic cathedral, though labyrinthine patterns are found with equal frequency as turf pathways or passages within gardens. While much scholarship has been spent on the interpretations of these woven graphical patterns, an exact understanding of their social or religious meaning is still clouded. The oft-cited notion of the church maze acting as a surrogate for pilgrimage is heatedly debated and compelling alternatives have been offered. Penelope Reed Doob offers tremendous insight into all aspects of the labyrinth, dismissing the pilgrimage metaphor as unfounded, favouring two more plausible alternatives, which combine to a form a third. As Doob states,

"So far, then, we have established two medieval reasons for placing

labyrinths in churches: as a sign of the enclosing cathedral's magnificence and the architect's genius, and as a sign of hell made extricable through the labors and unicursal footsteps of Christ-Theseus - a sign of redemption as well as a warning to those who do not follow

Christian doctrine." [2, pg 128]

While Doob's arguments continue with great depth and unravel a number of nuances and linkages between the labyrinth, other gothic patterns and tectonic expressions and their corresponding significance to medieval Christian worship, it is her discussions of the dualities of the labyrinth that find the most lasting resonance. Doob brings to light the two primary structures of the labyrinth, one being singular in path and requiring only persistence for navigation (unicursal), and the second being composed of multiple points of decision and direction, 
within which no one singular path exists (multicursal). While the unicursal model is most often employed as a symbol, both labyrinthine orders are equally latent with a number of diagrammatic dualities.

"A diagrammatic labyrinth paradoxically clarifies its own confusion; it holds structural design and the psychological tensions created by that design in perfect equilibrium, suggesting order in chaos, the purposefulness of apparent aimlessness, unity controlling multiplicity."

$[2, \mathrm{pg} 61]$

It is this sort of duality that makes the labyrinth eternal, for while the specific societal meanings have shifted and been displaced, the intrigue of labyrinthine space is perpetuated as a collection of physical simultaneities, of order and disorder, of lucidity and confusion, that beckons the darkest realms of the human consciousness to re-emerge as new and meaningful forms, with its most recent (and perhaps most spatially complete) manifestation within the immersive environments of digital gaming.

\section{Labyrinthine gaming}

To better grapple with these types of spaces, a bit of clarification is required, not only to offer the historical development of game space, but also the specific emphasis on first-person shooter immersion within the broader field of gaming strategies. Video gaming is commonplace within popular culture, both as part of a mature demographic whose adolescent lives served as the initiates to early video games and the subsequent generations who see these two-dimensional gaming methods as purely infantile novelties within the ever-more complex genre of computer gaming. While games such a Pong and Asteroids were seminal in the linage of game development, their conception of space is highly limited, perceived only as a two-dimensional graphic on the screen through which the player interacts. Though the many variants of these early games are more akin to the novelties of the multicursal labyrinths and mazes of childhood, even at this stage, the notion of labyrinth begins to become ingrained as a spatial strategy. Digital suggestions of walls and barriers, as well as maze-like configurations begin to appear, most notably within the series of games initiated with Pac-Man. This game, and the phenomenon that surrounded it, was all consuming as an interactive device. The infinite manifestations for negotiating the various levels produced more than occasional amusement. It founded a society of sorts, structured around the solving of the spatial puzzles (hints again back to the Cretan myth and Theseus' ball of thread), completing that soughtafter "high score" title through the graceful and fluid avoidance of menacing monsters (themselves quite endearing, appearing more like personified rag mops), and more importantly, the rejection of the exterior world.

The evolution of this early labyrinthine strategy has become quite farreaching, though one particular game offers a definitive link to the present day scenario of first-person shooter gaming platforms. The game Castle Wolfenstein was a rather clumsily composed maze game, complicated with a rudimentary storyline by which the maze was to be negotiated. In its most basic state, the 
narrative and maze worked in tandem, forcing the avatar or character to work through a series of adjoining but concealed chambers, avoiding or eliminating the computer sentinels that monitored each chamber, to arrive at the final destination. Upon eliminating the final sentinel (in the case of this World War II narrative, the fuehrer), the player would then need to retrace his steps out of the maze before being captured. The clumsiness of the movements and the restrictions of planar space offered little more than mild amusement for the digitally savvy adolescent, but its lineage proved far more fruitful. During the years between the creation of Castle Wolfenstein and its offspring Castle Wolfenstein $3 D$, the notion of first-person shooter gaming systems was sewn.

\section{Virtual immersion and the first-person shooter}

First-person shooter games are set on the premise of two basic strategies. On the more technical side, the logic of spatial development and representation is driven by the requirements of real-time animation, which requires a constant updating of the digital environment with each successive movement within the space. The second premise is more ingrained within the notion of the simulation, which is the fundamental point of reference, or position of the eyes. Nearly all games prior to the first-person shooter strategy had operated off of orthographic conventions of plan or elevation, defaulting to the overhead logic of Pac-Man, or elevational presentation of Donkey Kong. The first-person shooter strategy placed the player directly into the space, the screen becoming the virtual eyes of the character's head. This shift in strategy revealed an entirely new set of opportunities for gaming interaction - an enlightenment not dissimilar to the introduction of perspectival representation at the dawn of the renaissance.

Castle Wolfenstein 3D was the first foray into the FPS system, though it was essentially structured off of a similar narrative and spatial sequence as its predecessor. The player navigated a sequence of interlocked rooms and halls, eliminating all of the demons encountered along the way. While equally rudimentary in its spatial definition and means of navigation, Castle Wolfenstein $3 D$ (pioneered by id Software) found immense success with the spatial experience and simulation of movement and occupation. The player's movements and lines of sight were freed from the static and detached character of 2D game, allowing for the freedom to look, wander (and become further disoriented) within the spatial immersion. The player was in full control of their avatar, and thus the visual experience as presented to the screen. The 2D gaming methods, with their global and easily mapped presentation couldn't offer the same levels of concealment or intrigue because the absence of foreground and background. FPS suddenly revealed a navigable panorama of space to the player, to which the frontal view was privileged as seen through the eye, but with an equal awareness of the space to the player's back. The labyrinth was still the underlying means of ordering the spatial layout, though the perception of it was no longer planar, migrating away from the graphical mazes of childhood in favour of the spatial play of the Victorian garden mazes or folk crop-field mazes of the rural America. 
The current assortment of FPS games is far too numerous to attempt a discussion of labyrinth, spatial perception and sensory engagement for each platform, but it is possible to illuminate the commonalities and underpinnings of each, particularly as spatial representations of the labyrinth. As the forefront of this discussion should be the question of the FPS platform proper. The structure of Castle Wolfenstein $3 D$ was fundamentally narrative in both story and space, offering an itinerant experience that helped to organize the game play, which upon first pass was enthralling, but with successive play became predicable and ordinary. Drawn out of its foundation, however was a new pair of games, Doom and Doom 2, which offered an enhanced narrative play and spatial character, as well as introducing the possibilities of the multiplayer game play. While the original narrative game structure was limited to a single player, Doom allowed for the possibility of multiple players interacting within a single digital environment, either as team members working towards a common goal (a kin to the narrative model), or more seductively as equally matched opponents within an arena. No longer was the sole user reliant upon the artificial intelligence of the gaming engine to offer the competition, but rather the established competition through the direct interaction with another player, or group of players, distant in physical space, but immediately present within the digital realms of the FPS game environment. The tremendous success of arena games let to an entirely new method of game strategies, and a corresponding shift in the nature of the spatial environment within which the game play was to unfold. This method of gaming has quickly become the dominant product offering. While the narrative approach has remained as a consistent ingredient to the game systems, nearly all FPS games now offer arena play as a component if not the singular focus in games such as Quake Arena or Unreal Tournament.

This strategic shift within the industry has been ongoing, and runs parallel with equally provocative developments within the rendering engine itself. The level of realism and detail has become remarkable for the avatar's physical motion and control as well as the material environment in which they are immersed. Early version of FPS movement had the head/eye position locked into a singular horizontal plane, rendering the player unable to look up or down. New character mobility now offers flexible eye and head movement, as well as crouching, jumping, crawling, and any number of other specific body motions. Corresponding developments in spatial rendering have occurred, offering increased material detail, variation and model complexity and well as a host of other dynamic environmental characteristics, such as the play of light and shadow within a space, simulated material tactility (flowing fabrics), degrees of transparency and/or opacity, reflections, queued audio, as well as a number detailed refinements and subtleties, ranging from weather effects to material engagement and response.

\section{Piranesi's ghosts}

The foundations for these spatial strategies are still fundamentally rooted within the taxonomy of the labyrinth, but the spatial character has moved away from the 
simple limitations of planar movement. The new game environments are heavily labyrinthine, but more in the spirit of the classical narrative descriptions of the Cretan labyrinth, latent with interwoven paths and chambers, from which the most immediate product is of confusion and disorder, with no entrance disclosed and little or no exposure of a broader surrounding landscape. To this end, these digital spaces, so heavily interlocked with paths and discrete vantage points that appear inaccessible, bear a striking resemblance to the Imaginary Prisons etchings of Piranesi. This is not without surprise. Piranesi's visions of the dungeonous volumes graphically capture both the literary description of the classical labyrinth of myth while also providing and ideal perspectival template for the arena gaming. His conceptions are heavily imbedded with scalar shifts and dimensional ambiguities, of soaring passageways to and from undisclosed origins, all of which entice the eye to wander, but offer no means of orientation thus returning to the one of the many dualities of the labyrinth itself. As Marguerite Yourcenar noted in her essay "The Dark Brain of Piranesi,"

"No connoisseur of oneiric matters will hesitate a moment in the presence of these drawings evincing all the chief characteristics of the dream state: negation of time, incoherence of space, suggested levitation, intoxication of the impossible reconciled or transcended, terror closer to ecstasy than is assumed by those who analyze the visionary's creations from outside, absence of visible contact between the dream's parts or characters, and finally a fatal and necessary beauty." [3,pg 110]

Yourcenar's observations of the dream state that is incurred by the simple observation of Piranesi's prison sketches are both accurate and visually obvious. The prison sketches are saturated with various degrees of visual intrigue. Outside of the immediate spatial seductions, the spaces are latent with the suggestive devices of torture, along with the quintessential atmospheric characteristics that tapped into all of the senses. Yourcenar's notes these sensory seductions as well.

"Finally, this void is sonorous: each Prison is conceived as an enormous Ear of Dionysus. Just as in the Antiquities one hear the faint echo of an aeolian harp in the ruins, the rustling of the wind in the weeds and rushes, here the roused sense of hearing perceives a formidable silence in which the lightest footstep, the faintest sigh of the strange and diminutive strollers lost in these aerial galleries would echo from one end of the enormous structures to the other. " [3,pgs 111-112]

In fact, it is this sensory scintillation, this remarkable ability to transcend the purely visual and to touch on the fullest spectrum of experience that makes the relationship of Piranesi's prisons and the spatial layering of arena gaming so remarkably strong. If Piranesi's imagery creates a dizzying array of sensory suggestions through purely visual methods, the prison sketches are but mere hints of the verisimilitude revealed in immersive gaming. For while Piranesi's spatial movements cannot extend beyond the frozen moment of one specific view, the immersive game environment allows the bewildered participant the freedom of movement, the opportunity to explore the fullest spatial rapture that Piranesi was surely dreaming of. If the stories of the origin of Piranesi's 
Imaginary Prisons emerging from illness-induced delusions is accurate, then the first-person shooter game environment is this dreamscape (or nightmare) made physically manifest, albeit within the confines of the digital realm. Yet this digital constriction hardly limits the physical experience. Just as a dog dreaming may physically exhibit the chase envisioned with twitching legs and muffled barks, the participant within the game environment responds to the spatial experiences with distinct physical reaction. No longer does the player remain passive to the stimuli, but is now fully engaged, bobbing and weaving in vain attempts to avoid the various threats of the opponent which is often undisclosed, and almost entirely anonymous.

It is this very character of the game, a re-emergence of sorts of the dualisms expressed in the classical interpretations of the labyrinth and its many narratives, that have drawn their spatial inspiration from Piranesi's space (though perhaps unknowingly). The player within the game is simultaneously protagonist and antagonist within the unfolding story, acting as a direct assault to their opponents from the singularly framed perspectival screen image, fully conscious of their equal and omnipresent position as the target. The soaring spaces and multiple vantage points of Piranesi's sketches provide fertile ground, offering numerous points of concealment and paths for covert movement from which to gaze upon the bounded combat theatre. In fact, at the heights of the game, when the dynamic play rises to levels of frantic movement and evasion, exaggerating the visual enrapture provided by the multitude of passageways and portals, the interwoven volumes that repeatedly turn inward upon themselves, enticing the player into constant motion both visual and physical within a sequence of spaces with no entry and no exit. Yourcenar notes an equal trait in the Prison sketches, which find near-exact replications in the spatial containments of the arena.

"Often the arch of a vault in the upper part of the image conceals the top steps of a staircase or the end of a ladder, suggesting heights still loftier than those of the steps and rungs visible; the hint of another staircase plunging lower than the level on which we are standing warns that this abyss is also to be extended beyond the plate's lower margin; the suggestion grows even more specific when a lantern hung almost on a level with the same margin confirms the hypothesis of invisible black depths below. Moreover, the artist succeeds in convincing us that this disproportionate hall is hermetically sealed, even if the face of the cube we never see because it is behind us. " [3, pg 113]

It is this notion of the hermetical seal, of absolute containment that reaffirms the labyrinthine character. The early labyrinths, as both physical and metaphorical operations, are in a constant state of simultaneous expressions/simulations, to which Piranesi and the immersive game environments prove a natural extension. The classical labyrinths of antiquity no longer survive, though their impenetrable brilliances and spatial conundrums are well documented throughout any number of historical texts. Yet, when reading the verbal descriptions, the immediate visual depiction aligns quickly to Piranesi, which in turn flips from the perspectival suggestions of his two-dimensional plates, to the virtual realities through which gaming arenas have found emerged. To be sure, the metaphorical 
significance of the labyrinthine story still unfolds within the game systems, but its hints at morality and conscience are quickly deflated to the most grotesque levels of amusement. Thus it is the physical construct of the metaphor that has been maintained, as if in some subconscious acknowledgement that the meaning of such stories, or their redemption within the accelerated visual culture of modern society, must be presented within the notion of labyrinthine space itself.

\section{Closing thoughts}

When looking to the nature of society and its on-going infatuation with the digital realm, it is of little wonder that the Piranesian hauntings within the gaming world are so easily detected. Equally, the application of the labyrinth as a spatial construct for these games seems is not only a convenient and logical extension of the amusement garden mazes, but also hints at a perpetuation of fascinations of the darker realms of the human imagination. Though a great deal of time and effort has been spent on the examination of the physical aspects of the construct, relieving the burdensome chores of exploring of societal meanings of these forms, this sort of reading needs receive a nod of recognition, even if it is somewhat cursory.

The labyrinthine fascinations of the antiquities occurred as a two-fold event in which the physical manifestation was inextricably a part of the corresponding myth. To this end, the physical system of labyrinth, whether as a spatial condition itself or as a graphical representation, was impregnated with an understood societal meaning - to speak of the Chartres labyrinth as simply a geometrical weaving stripped of symbolic significance is absurd. Even if the modern understandings of its significance must rely upon certain approximations or historical interpretations, the knowledge and understanding of society in the classical periods, or more so in the medieval periods make clear that the labyrinthine form was latent with meanings and rituals. Yet, the discussion of game space and its borrowing of the labyrinthine logic suggest that these physical forms have been stripped of this meaning, re-occupied as if the labyrinth is simply a shell for the purest sake of novelty and distraction. However, if the fullest understanding of the labyrinth has perpetuated as a form from the earliest ages of the antiquities, repeated in the imagery of Piranesi and retold in the gaming environment, does this not imply an equal but often overlooked social significance? Yourcenar on again provides some insight into this avenue through Piranesi's visions:

"We cannot help thinking of our theories, our systems, our magnificent and futile mental constructions in whose corners some victim can always be found crouching. If these Prisons, for so long relatively neglected, now attract the attention of a modern public as they do, it is perhaps not only, as Aldous Huxley has said, because this masterpiece of architectural counterpoint prefigures certain conceptions of abstract art but above all because this world, factitious and yet grimly real, claustrophobic and yet megalomaniacal, cannot fail to remind us of the one in which modern humanity imprisons itself deeper every day, and whose mortal dangers we are beginning to recognize." [3, pgs 120-121] 
If, as Yourcenar suggests, the relevance of Piranesi's Imaginary Prisons resounds with some prophetic visual speculation of the modern built world, then perhaps the games and the spatial escapism that they offer is equally revealing. The storylines and gaming strategies seem to revolve around the grotesque, often situated within contexts so grim as to align, if not surpass the bestial narratives of the Minotaur, or of Dante's descent into the Inferno. This fixation upon the debased, when combined with the excessive dynamism of the game play and the remarkably charged and disorienting chambers through which it occurs, indicates that the character and stimulation of these virtual realms is far more rewarding than the physical environments that the players can actually engage. To this end, the simultaneity of experience, the otherworldly seductions that immersive gaming environments perhaps offer some level of debased lucidity to a world that is otherwise mired its own banality.

\section{References}

[1] Eco, Umberto, In the Name of the Rose, Harcourt Brace \& Company, New York, 1983. pg 217.

[2] Doob, Penelope Reed, The Idea of the Labyrinth from Classical Antiquity through the Middle Ages, Cornell University Press, Ithaca, 1990.

[3] Yourcenar, Marguerite, The Dark Brain of Piranesi and Other Essays, trans. By Richard Howard. Farrar, Straus, and Giroux, Inc., New York, 1980 (translation copy). 